\title{
REPRESENTATIONS OF MINIMALLY ALMOST PERIODIC GROUPS
}

\author{
ALAIN VALETTE \\ (Received 9 April 1985) \\ Communicated by W. Moran
}

\begin{abstract}
For any group $G$, we introduce the subset $S(G)$ of elements $g$ which are conjugate to $g^{2^{k}}, g^{3^{k}}, g^{4^{k}}, \ldots$ for some positive integer $k$. We show that, for any bounded representation $\pi$ of $G$ and any $g$ in $S(G)$, either $\pi(g)=1$ or the spectrum of $\pi(g)$ is the full unit circle in $C$. As a corollary, $S(G)$ is in the kernel of any homomorphism from $G$ to the unitary group of a post-liminal $C^{*}$-algebra with finite composition series.

Next, for a topological group $G$, we consider the subset of elements approximately conjugate to 1 , and we prove that it is contained in the kernel of any uniformly continuous bounded representation of $G$, and of any strongly continuous unitary representation in a finite von Neumann algebra.

We apply these results to prove triviality for a number of representations of isotropic simple algebraic groups defined over various fields.
\end{abstract}

1980 Mathematics subject classification (Amer. Math. Soc.): 22 D 12; secondary 20 G 15, 46 L 05.

\section{Introduction}

Let $G$ be a topological group; the intersection $n(G)$ of the kernels of the finite-dimensional continuous unitary representations of $G$ is the von Neumann kernel of $G$; this closed normal subgroup of $G$ can be completely characterized when $G$ is locally compact and connected (see [13], [14]). $G$ is said to be minimally almost periodic (m.a.p.) if $G=n(G)$, i.e. if $G$ has no non-trivial finite-dimensional continuous unitary representations. In their paper [11], von Neumann and Wigner obtained the following useful sufficient condition for an element $g$ in $G$ to belong to $n(G)$ : assume that there exists a function $f$ from the set $\mathbb{N}_{0}$ of

(C) 1986 Australian Mathematical Society $0263-6115 / 86 \$ A 2.00+0.00$ 
positive integers to itself, such that, for any $n$ in $\mathbb{N}_{0}, n$ divides $f(n)$ and $g$ is conjugate to $g^{f(n)}$ inside $G$; then $g$ belongs to $n(G)$, when $G$ is endowed with the discrete topology.

In Section 1 of this paper, we consider the subset $S(G)$ of elements in $G$ for which the function $f$ above can be taken of the form $f(n)=n^{k}$, for some $k$ in $\mathbb{N}_{0}$. The interest of this case comes from the example of $\mathrm{SL}_{2}(k)$, where $k$ is a field of characteristic 0 ; for any $a$ in $k$ and any $\lambda$ in $k^{\times}$, the multiplicative group of $k$, we have:

$$
\left(\begin{array}{cc}
\lambda & 0 \\
0 & \lambda^{-1}
\end{array}\right)\left(\begin{array}{cc}
1 & a \\
0 & 1
\end{array}\right)\left(\begin{array}{cc}
\lambda^{-1} & 0 \\
0 & \lambda
\end{array}\right)=\left(\begin{array}{cc}
1 & \lambda^{2} a \\
0 & 1
\end{array}\right)
$$

In particular, taking for $\lambda$ a positive integer $n$, we see that $g=\left(\begin{array}{ll}1 & a \\ 0 & 1\end{array}\right)$ is conjugate to $g^{n^{2}}$, for any $n$. We will extend the von Neumann and Wigner result by showing that, for any $g$ in $S(G)$ and any bounded representation $\pi$ of $G$ on a (complex) Banach space, the following alternative holds: either $\pi(g)=1$ or the spectrum of $\pi(g)$ is the unit circle $S^{1}$ in C. As a corollary, we will see that isotropic simple algebraic groups over $k$ (of characteristic 0 ) are m.a.p. in a very strong sense: roughly speaking, we will show that any homomorphism from such a group to the unitary group of a post-liminal $C^{*}$-algebra with finite composition series, is trivial (see Corollary 2 and Theorem 2 for the precise statement).

In Section 2, we consider, for a topological group $G$, two classes of continuous representations which are "close to" finite-dimensional ones, namely uniformly continuous bounded representations on a Banach space, and strongly continuous unitary representations in a finite von Neumann algebra (we refer to [12] for terminology). To explain what we have in mind, let us mention the following result, proved by Singer [16] for unitary representations, and extended by Gurarie [3] to more general representations: a connected Lie group $G$ admits a faithful uniformly continuous bounded representation if and only if $G$ is the direct product of a (finite-dimensional) real vector space and a compact Lie group. This in turn is equivalent to the existence of a faithful strongly continuous unitary representation of $G$ in a finite von Neumann algebra (a result due to Kadison and Singer [8, p. 64]). Moreover, it was shown by Kallman [6] that the disintegration of any uniformly continuous unitary representation of a locally compact connected group involves a measure which is compactly supported in the set of finite-dimensional irreducible unitary representations. Finally, there are results saying that, roughly speaking, irreducible uniformly continuous representations are finite-dimensional (see [3], [4], [16]). We shall give a unified proof of the following result: if $k$ is a non-discrete locally compact field (of any characteristic), and if $G_{k}$ is the group of $k$-rational points of some isotropic simple algebraic group defined over $k$, endowed with its natural locally compact topology, then any strongly continuous unitary representation of $G_{k}$ in a finite von Neumann 
algebra and any uniformly continuous bounded representation of $G_{k}$ on a Banach space factorize through a compact abelian group. (Moreover, the second assertion is even true without the boundedness assumption if $k \neq \mathbb{R}, \mathbb{C}$.) The results are related to the existence in $G_{k}$ of a "large" (we quote Tits [17, p.314]) normal subgroup $G_{k}^{0}$ which is m.a.p. Our proof was motivated by the proof of von Neumann and Segal [10] of the fact that strongly continuous unitary representations of a simple non-compact Lie group in a finite von Neumann algebra are trivial; our idea is to consider elements $g$ of a topological group $G$ which are approximately conjugate to 1 , i.e. such that the closure of the conjugacy class of $g$ contains the identity 1 of $G$. For example, in $\mathrm{SL}_{2}(k)$, the element $g=\left(\begin{array}{ll}1 & a \\ 0 & 1\end{array}\right)$ has this property, as we see from formula (*) by letting $\lambda$ tend to 0 in $k$. The proof will proceed by introducing a class $\mathscr{C}$ of topological groups such that, if $H$ belongs to $\mathscr{C}$, and if $G$ is any topological group, the set of elements in $G$ which are approximately conjugate to 1 is in the kernel of any continuous homomorphism $G \rightarrow H$.

\section{The set $S(G)$}

We recall that, for any group $G$, we have defined $S(G)$ to be the set of $g$ 's in $G$ such that, for some $k$ in $\mathbb{N}_{0}, g$ is conjugate to $g^{n^{k}}$ for any $n$ in $\mathbb{N}_{0}$. For any element $x$ in a Banach algebra $A$, we denote by $\operatorname{sp} x$ (respectively $r(x)$ ) the spectrum (respectively spectral radius) of $x$.

Proposition 1. Let $A$ be a unital Banach algebra, and let $x$ be an element in $S\left(A^{-1}\right)$. Then either $\operatorname{sp} x=\{1\}$ or $\operatorname{sp} x=S^{1}$.

Proof. First, we claim that $\operatorname{sp} x$ is contained in $S^{1}$. Indeed, since $x$ belongs to $S\left(A^{-1}\right)$, there exists a $k$ in $\mathbb{N}_{0}$ such that

$$
\operatorname{sp} x=\operatorname{sp}\left(x^{n^{k}}\right)=(\operatorname{sp} x)^{n^{k}} \quad \text { for any } n \text { in } \mathbb{N}_{0} .
$$

So, if some $z$ in $\operatorname{sp} x$ is such that $|z| \neq 1$, then $\operatorname{sp} x$ contains the sequence $\left(z^{n^{k}}\right)_{n \in \mathbf{N}_{0}}$, which contradicts the compactness of $\operatorname{sp} x$ in $\mathbf{C}^{\times}$. Now, we identify $S^{1}$ with $\mathbb{R} / \mathbb{Z}$ by $s \rightarrow \exp (2 \pi i s)$. The result then follows from the next lemma.

LemMa 1. Let $X$ be a non-empty closed subset of $\mathbb{R} / \mathbb{Z}$, such that $n^{k} X=X$ for any $n$ in $\mathbb{N}_{0}$. Then either $X=\{0\}$ or $X=\mathbb{R} / \mathbb{Z}$.

Proof. We use several steps. 
Step 1. If $X$ is finite, then $X=\{0\}$. Indeed, let $\sigma$ be the permutation of $X$ defined by $\sigma(x)=2^{k} x(x \in X)$. Let $m$ be the cardinal of $X$; iterating $m$ ! times the preceding relation (although the order of $\sigma$ in the symmetric group Sym $m$ would suffice), we get

$$
x=\left(2^{k}\right)^{m !} x \text { i.e. } 0=\left(2^{k \cdot m !}-1\right) x .
$$

So, for some positive integer $l, X$ is contained in the set $\Omega$, of elements of order $l$ in $\mathbb{R} / \mathbf{Z}$. Consequently $X=l^{k} X$ is contained in $l^{k} \Omega_{l}=\{0\}$.

Step 2. If $X$ admits 0 as a limit-point, then $X=\mathbb{R} / \mathbb{Z}$. Indeed, let $\left(x_{n}\right)_{n \in \mathbb{N}_{0}}$ be an injective sequence tending to 0 in $\mathbb{R} / \mathbb{Z}$. Identify $\mathbb{R} / \mathbb{Z}$ with the interval $[0,1[$; then $\left(x_{n}\right)$ is a sequence in $[0,1[$ having at most two limit points, 0 and 1 . Assume that 0 is such a limit-point; then there is a subsequence $\left(y_{n}\right)_{n \in \mathbb{N}_{0}}$ tending to 0 . Denoting by $[x]$ the integer part of the real number $x$, we consider the following sequence:

$$
y_{1}, 2^{k} y_{1}, 3^{k} y_{1}, \ldots,\left[y_{1}^{-1 / k}\right]^{k} y_{1}, y_{2}, 2^{k} y_{2}, \ldots,\left[y_{2}^{-1 / k}\right]^{k} y_{2}, y_{3}, \ldots
$$

This sequence is clearly contained in $X$. Now, consider the sequence:

$$
\begin{gathered}
y_{1}^{1 / k}, 2 y_{1}^{1 / k}, 3 y_{1}^{1 / k}, \ldots,\left[y_{1}^{-1 / k}\right] y_{1}^{1 / k}, y_{2}^{1 / k}, 2 y_{2}^{1 / k}, \ldots,\left[y_{2}^{-1 / k}\right] y_{2}^{1 / k}, \\
y_{3}^{1 / k}, 2 y_{3}^{1 / k}, \ldots,\left[y_{3}^{-1 / k}\right] y_{3}^{1 / k}, y_{4}^{1 / k}, \ldots,
\end{gathered}
$$

which is dense in $\left[0,1\left[\right.\right.$ since $\left(y_{n}^{1 / k}\right)$ tends to 0 . But (2) is obtained by taking the $k$ th root of each term in (1), so (1) itself is dense. All this shows that $X$ is dense in $\mathbb{R} / \mathbb{Z}$, and this concludes our proof in the case where 0 is a limit-point of $\left(x_{n}\right)$. The case where 1 is a limit-point can be reduced to the previous one by identifying $\mathbb{R} / \mathbb{Z}$ with $[-1,0[$ instead of $[0,1[$. This concludes the second step.

Step 3. We now prove the lemma itself. Assume that $X$ contains some irrational number $\boldsymbol{\vartheta}$. Then $X$ contains the sequence $\left(n^{k} \boldsymbol{\vartheta}\right)_{n \in \mathbb{N}_{0}}$ which, by van der Corput's theorem (see [7, Theorem 3.2]), is uniformly distributed, hence dense, in $\mathbb{R} / \mathbb{Z}$. So $X=\mathbb{R} / Z$ in this case. It remains to show that if $X$ is contained in $\mathbb{Q} / \mathbb{Z}$, then necessarily $X=\{0\}$. Assume the contrary; then, by step 1 of the proof, $X$ is infinite, so by compactness $X$ contains at least one limit-point $x$. Since $X$ is rational, we find a positive integer $l$ such that $0=l x$ in $\mathbb{R} / \mathbb{Z}$. So $0=l^{k} x$ is a limit-point of $X$ as well, and by step 2 of the proof, we have $X=\mathbb{R} / \mathbb{Z}$, a contradiction. This proves Lemma 1 together with Proposition 1.

Remark 1. Step 1 of the above proof is essentially Lemma 1 of [11]. This step suffices to show that, for any group $G$, the set $S(G)$ is contained in $n(G)$. More generally, for any field $K$ and any group $H$ of diagonalizable matrices in $\mathrm{GL}_{n}(K)$, the set $S(G)$ is contained in the kernel of any homomorphism $G \rightarrow H$. 
We denote by GL( $E)$ the group of bounded invertible operators on the Banach space $E$, and by $\mathrm{GL}_{0}(E)$ the subgroup of those operators that are scalar modulo compacts.

Lemma 2. Let $E$ be a Banach space; if $T \in \mathrm{GL}(E)$ is such that $\operatorname{sp} T=\{1\}$ and $\sup \left\|T^{n}\right\|<\infty$, then $T=1$.

Proof. There are two cases where this lemma is well known: if either $E$ is a Hilbert space (then the representation of $\mathbf{Z}$ defined by $T$ can be unitarized, by amenability of $\mathbb{Z}$ ), or $T$ is an isometry on $E$ (then the lemma is just [12, 8.1.11]). For the general case, we find by holomorphic functional calculus a quasi-nilpotent operator $H$ such that $e^{H}=T$. Then a simple interpolation argument shows that the uniformly continuous representation $t \rightarrow e^{t H}$ of $\mathbb{R}$ is bounded; by Lemma 1 of [3], we have $H=0$, i.e. $T=1$.

From Proposition 1 and Lemma 2, we deduce

THEOREM 1. Let $G$ be a group, and let $\pi$ be a bounded representation on some Banach space. Then, for any $g$ in $S(G) \backslash \operatorname{Ker} \pi$, we have $\operatorname{sp} \pi(g)=S^{1}$ and $\|\pi(g)-1\| \geqslant 2$, with equality if $\pi$ is unitary.

Proof. To prove the inequality, simply notice that

$$
2=r(\pi(g)-1) \leqslant\|\pi(g)-1\|
$$

because sp $\pi(g)=S^{1}$. The other statements are clear.

COROLlary 1. If $\pi$ is a bounded representation of $G$ whose image is contained in $\mathrm{GL}_{0}(E)$, then $S(G)$ is contained in $\mathrm{Ker} \pi$.

This follows from Theorem 1 and from the fact that $\operatorname{sp} \pi(g)$ is countable.

COROllary 2. Let $A$ be a unital post-liminal $C^{*}$-algebra admitting a finite composition series $0=I_{0} \triangleleft I_{1} \triangleleft \cdots \triangleleft I_{n}=A$ such that $I_{i+1} / I_{i}$ is liminal for $i=$ $0,1, \ldots, n-1$. Let $\mathscr{U}(A)$ be the unitary group of $A$. Then, for any group $G$, the set $S(G)$ is contained in the kernel of any homomorphism $G \rightarrow \mathscr{U}(A)$.

Proof. (For general background on post-liminal $C^{*}$-algebras and composition series, see [12].) We have to show that $S(\mathscr{U}(A))=\{1\}$. Assume, by contradiction, that there exists some $u \neq 1$ in $S(\mathscr{U}(A))$. Denoting by $\tilde{I}_{i}$ the unital $C^{*}$-sub-algebra generated by $I_{i}$, we see that, for some $i>0$, the unitary $u$ belongs to $\tilde{I}_{i}$ but not to $\tilde{I}_{i-1}$; let $\dot{u}$ be the image of $u$ in $\tilde{I}_{i} / I_{i-1}(\dot{u} \neq 1)$, and let $\pi$ be an irreducible 
representation of $\tilde{I}_{i} / I_{i-1}$ on some Hilbert space $\mathscr{H}_{n}$, such that $\pi(\dot{u}) \neq 1$. Since $I_{i} / I_{i-1}$ is liminal, $\pi(\dot{u})$ belongs to $\mathrm{GL}_{0}\left(\mathscr{H}_{\pi}\right)$, but this contradicts Corollary 1 .

We conclude this section by giving some examples to which the preceding results apply.

Proposition 2. Let $k$ be a field of characteristic 0 , and let $H$ be a subgroup of $\mathrm{GL}_{m}(k)$ admitting a cyclic vector $x_{0}$ in $k^{m}$, and such that, for any $n \in \mathbb{N}_{0}$, there is an $h_{n} \in H$ satisfying $h_{n}\left(x_{0}\right)=n x_{0}$. Let $G$ be the semi-direct product $k^{m} \rtimes H$, and let $\pi$ be a representation of $G$ satisfying the assumptions of either Corollary 1 or Corollary 2. Then $\pi$ factorizes through $\mathrm{H}$.

Proof. It is enough to show that the subgroup generated by $S(G)$ contains $k^{m}$. Clearly, for any $\lambda \in k^{\times}$and any $h \in H$, the element $h\left(\lambda x_{0}\right)$ is in $S(G)$; by cyclicity of $x_{0}$, we may select a basis $x_{1}, \ldots, x_{m}$ inside the orbit $H x_{0}$, and the preceding argument shows that any linear combination of the $x_{i}$ 's, i.e. any element of $k^{m}$, belongs to the subgroup generated by $S(G)$.

An important application of this result is given by the " $a x+b$ " group of $k$, i.e. the semi-direct product $k \rtimes k^{\times}$. In particular, any finite-dimensional unitary representation of this group factorizes through $k^{\times}$(for a locally compact non-discrete $k$ and a continuous representation, this follows immediately from Mackey's theory [8]).

THEOREM 2. Let $G$ be an isotropic simple algebraic group defined over some field $k$ of characteristic 0 . Let $G_{k}$ be its group of $k$-rational points, and $G_{k}^{0}$ be the subgroup generated by all unipotent $k$-subgroups which are split over $k$. Let $\pi$ be a representation of $G_{k}$ satisfying the assumptions of either Corollary 1 or Corollary 2. Then $\pi$ factorizes through $G_{k} / G_{k}^{0}$.

The group $G$ is isotropic if it admits a split torus of positive dimension (over $k$ ). In characteristic $0, G_{k}^{0}$ may be defined more simply as the subgroup generated by all unipotent elements of $G_{k}$ (see $[1,6.2]$ ). The structure of $G_{k} / G_{k}^{0}$ is discussed in $[17,1.4]$. A conjecture of Kneser and Tits asserts that this group is always abelian, and is trivial if $G$ is simply connected over $k$ (this is known if $G$ admits a Borel subgroup defined over $k[1,6.6],[17,1.4])$. If $G$ is a classical group, the structure of $G_{k} / G_{k}^{0}$ is given explicitly in [2]. In any case, $G_{k}^{0}$ is Zariski-dense in $G$ ([17, 3.2(20)]).

Following Howe and Moore [5], we say that a one-parameter subgroup in $G_{k}$ is a non-trivial algebraic homomorphism $\beta: k_{\text {add }} \rightarrow G_{k}$, and that a one-parameter subgroup $\beta$ is of Jacobson-Morosov type if there exists an homomorphism $\mathrm{SL}_{2}(k) \rightarrow G_{k}$ which coincides with $\beta$ when restricted to the subgroup $k_{\text {add }}$ of upper strictly triangular matrices (possibly after a reparametrization of $\beta$ ). It is 
clear from formula $(*)$ in the introduction that, in characteristic 0 , the union of all one-parameter subgroups of Jacobson-Morosov type is contained in $S\left(G_{k}\right)$.

Proof of Theorem 2. It suffices to show that the (normal) subgroup of $G_{k}$ generated by $S\left(G_{k}\right)$ contains $G_{k}^{0}$. But, according to a theorem of Tits [17, 1.1], the group $G_{k}^{0}$ is simple modulo its centre. So it is enough to prove that $G_{k}^{0}$ contains one-parameter subgroups of Jacobson-Morosov type. This follows from root theory: to any restricted root $\alpha$ of $G$ (such an $\alpha$ does exist, for $G$ is isotropic), one associates a non-trivial homomorphism $\beta: \mathrm{SL}_{2}(k) \rightarrow G_{k}^{0}$ (see $[17,3.1(13)$ and 3.3]). This concludes the proof.

REMARK 2. As a consequence, we see that any finite-dimensional unitary representation of $G_{k}$ factorizes through $G_{k} / G_{k}^{0}$. Several variants of this result can be found in the literature, especially in Borel and Tits' paper [1] (for example, this is a consequence of $[1,10.3]$ in the case where $k$ is not a subfield of $\mathbb{C}$; also, a very particular case of $\left[1\right.$, Theorem A] shows that there is no homomorphism $G_{k}^{0} \rightarrow$ $\mathrm{SU}(n)$ with Zariski-dense image).

Remark 3. Theorem 2 implies the fact that connected simple non-compact Lie groups are m.a.p. in the discrete topology. Taking Remark 1 into account, we see that a finite-dimensional representation of such a group by normal operators is trivial as well. This is a particular case of a result of Sherman [15].

REMARK 4. As a consequence of Theorem 1, we see that, for any $g$ belonging to a one-parameter subgroup of Jacobson-Morosov type in $G_{k}$, and any unitary representation $\pi$ of $G_{k}$ such that $\pi(g) \neq 1$, the spectrum of $\pi(g)$ is $S^{1}$. For $k=\mathbb{R}$ and strongly continuous representations $\pi$ not containing the trivial representation, there is a much more precise result due to Moore [9, Theorem 2], which completely classifies the unitary type of $\pi(g)$.

\section{Elements approximately conjugate to 1}

We say that a topological group $G$ belongs to the class $\mathscr{C}$ if there exists a function $\varphi$ on $G$ which is central (i.e. constant on conjugacy classes), continuous at 1 , and such that $\varphi(g)=0$ if and only if $g=1$.

The following proposition is an immediate consequence of the definition.

Proposition 3. Let $G, H$ be topological groups, and let $\beta$ : $G \rightarrow H$ be a continuous homomorphism. If $H$ belongs to class $\mathscr{C}$, then the set of elements approximately conjugate to 1 in $G$ is contained in $\operatorname{Ker} \beta$. 
This proposition is exemplified by the following result.

Proposition 4. The following groups belong to class $\mathscr{C}$.

(i) The unitary group of a finite von Neumann algebra $M$, endowed with the strong topology.

(ii) Any bounded subgroup of $\mathrm{GL}(E)$, endowed with the norm topology (where $E$ is a Banach space).

Proof. (i) Let $\tau$ be any faithful, finite, normal trace on $M$; for $g$ in $\mathscr{U}(M)$, define $\varphi(g)=\tau(1-g)$; then $\varphi$ is central, it is strongly continuous (by [12, 3.6.4]), and the third condition is proved as in [10] (using the fact that, if $\operatorname{Re}(g)$ is the real part of $g$, then $1-\operatorname{Re}(g)$ is a positive element).

(ii) Define $\varphi(g)=r(g-1)$; the conclusion follows from Lemma 2 .

From this, we immediately deduce

COROLlaRY 3. For any topological group $G$, the set of elements approximately conjugate to 1 in $G$ is contained in $n(G)$.

Let us give examples where these results apply

Proposition 5. Let $k$ be a non-discrete locally compact field of any characteristic, and let $H$ be a closed subgroup of $\mathrm{GL}_{m}(k)$. Assume that there exist a vector $x_{0}$ in $k^{m}$, cyclic for $H$, and for any $\lambda \in k^{\times}$, an element $h_{\lambda} \in H$ such that $h_{\lambda}\left(x_{0}\right)=\lambda x_{0}$. Then any continuous homomorphism from the semi-direct product $G=k^{m} \rtimes H$ to a group of class $\mathscr{C}$ factorizes through $H$.

This is proved exactly like Proposition 2. In particular, it applies to the " $a x+b$ " group of $k$.

THEOREM 3. Let $k$ be a non-discrete locally compact field, and let $G, G_{k}, G_{k}^{0}$ be as in Theorem 2. Endow $G_{k}$ with its natural locally compact topology. Any continuous homomorphism from $G_{k}$ to a group of class $\mathscr{C}$ factorizes through $G_{k} / G_{k}^{0}$, a compact abelian group.

Note that $G$ is isotropic if and only if $G_{k}$ is non-compact in its locally compact topology. Results of Borel-Tits $[1,6.14-15]$ assert that $G_{k}^{0}$ is closed in $G_{k}$, and that $G_{k} / G_{k}^{0}$ is compact abelian. Moreover, if $k=\mathbb{R}, G_{k}^{0}$ coincides with the topological connected component of the identity in $G_{k}$. 
Proof of Theorem 3. We begin with $G=\mathrm{SL}_{2}$; but we saw in the introduction that $\mathrm{SL}_{2}(k)$ is generated by elements approximately conjugate to 1 . For a general $G$, as in Theorem 2 we associate to any restricted root a non-trivial continuous homomorphism $\mathrm{SL}_{2}(k) \rightarrow G_{k}^{0}$. So $G_{k}^{0}$ contains non-central elements which are approximately conjugate to 1 , and the simplicity of $G_{k}^{0}$ modulo its centre allows one to conclude.

REMARK 5. The preceding result shows that any uniformly continuous bounded representation of $G_{k}$ factorizes through $G_{k} / G_{k}^{0}$ (and the proof shows that $G_{k}^{0}$ is m.a.p.). In characteristic 0 , it is possible to deduce these results from Theorem 1; indeed, let $\beta: k_{\text {add }} \rightarrow G_{k}^{0}$ be a continuous one-parameter subgroup of JacobsonMorosov type. If $\pi$ is a non-trivial bounded representation of $G_{k}^{0}$, then by Theorem 1, for any $s \in k^{\times}: 2 \leqslant\|1-\pi(\beta(s))\|$. So, letting $s$ tend to 0 in $k$, we see that $\pi$ cannot be uniformly continuous. Note that for $k=\mathbb{R}, \mathbf{C}$, one might also reduce the whole problem to a finite-dimensional situation by using the fact that any uniformly continuous representation of $G_{k}^{0}$ is the direct sum of finite-dimensional irreducible representations (see [4, Proposition 4]; this is proved using Weyl's unitary trick).

Corollary 4. If $k \neq \mathbb{R}, \mathbb{C}$, and if $G, G_{k}, G_{k}^{0}$ are as above, then any uniformly continuous representation of $G_{k}$ factorizes through $G_{k} / G_{k}^{0}$ (without any boundedness assumption).

Proof. As in Theorem 3, it is enough to give the proof for $G=\mathrm{SL}_{2}$. But since $k$ is distinct from $\mathbb{R}$ and $\mathbb{C}, k$ contains a valuation ring $\omega$, and $S L_{2}(\omega)$ is a maximal compact subgroup of $S L_{2}(k)$; so the restriction of any uniformly continuous representation $\pi$ to $\mathrm{SL}_{2}(\omega)$ is bounded, and since any element $g=$ $\left(\begin{array}{ll}1 & a \\ 0 & 1\end{array}\right), a \in \omega$, is approximately conjugate to 1 in $\mathrm{SL}_{2}(k)$, we see by Proposition 3 and 4 that $g$ belongs to $\operatorname{Ker} \pi$ (see [4, Proposition 5] for a different proof).

Concerning finite-dimensional representations, the preceding corollary has some overlap with Théorème $(\mathrm{A})$ in Borel-Tits [1]. Indeed, it follows from this result that $G_{k}^{0}$ has no embedding in $\mathrm{GL}_{n}(\mathrm{C})$ if char $k \neq 0$, even if there is no continuity condition. On the other hand, in characteristic 0 , our corollary is in a certain sense "best possible", since there are plenty of embeddings of $p$-adic fields into $C$ giving rise to discontinuous representations of $G_{k}^{0}$.

To conclude, we mention that, for finite-dimensional unitary representations of $G_{k}$ (char $k=0$ ), our Theorem 2 seems to be stronger than Theorem 3, since there are no continuity assumptions involved in Theorem 2 . However, another result of Borel-Tits $[1,9.1]$ shows that any homomorphism from $G_{k}$ to a compact group is necessarily continuous; this extends an old result of van der Waerden [18] in the case $k=\mathbb{R}$. 


\section{Acknowledgement}

The author thanks Pierre de la Harpe for pointing out a number of useful references.

\section{References}

[1] A. Borel et J. Tits, 'Homomorphismes "abstraits" de groupes algébriques simples', Ann. of Math. 98 (1973), 499-573.

[2] J. Dieudonné, La géométrie des groupes classiques, 2nde édition (Springer-Verlag, Berlin and New York, 1963).

[3] D. Gurarie, 'Banach uniformly continuous representations of Lie groups and algebras', $J$. Funct. Anal. 36 (1980), 401-407.

[4] D. Gurarie, 'Banach uniformly continuous representations of locally compact groups', preprint, 1979.

[5] R. E. Howe and C. C. Moore, 'Asymptotic properties of unitary representations', J. Funct. Anal. 32 (1979), 72-96.

[6] R. V. Kallman, 'A characterization of uniformly continuous unitary representations of connected locally compact groups', Michigan Math. J. 16 (1969), 257-263.

[7] L. Kuipers and H. Niederreiter, Uniform distribution of sequences (Texts \& Monographs in Pure \& Applied Math., J. Wiley, New York, 1974).

[8] G. W. Mackey, The theory of unitary group representations (Chicago Lecture Notes in Math., Univ. of Chicago Press, 1976).

[9] C. C. Moore, 'Restrictions of unitary representations to subgroups, and ergodic theory' (Lecture Notes in Physics, 6, Springer-Verlag, Berlin and New York, 1970, pp. 1-36).

[10] J. von Neumann and I. E. Segal, 'A theorem on unitary representations of semi-simple Lie groups', Ann. of Math. 52 (1950), 509-517.

[11] J. von Neumann and E. P. Wigner, 'Minimally almost periodic groups', Ann. of Math. 41 (1940), 746-750.

[12] G. K. Pedersen, $C^{*}$-algebras and their automorphism groups (London Math. Soc. Monographs, 14, Academic Press, New York, 1979).

[13] S. Rothman, 'The von Neumann kernel and minimally almost periodic groups', Trans. Amer. Math. Soc. 259 (1980), 401-421.

[14] S. Rothman and H. Strassberg, 'The von Neumann kernel of a locally compact group', $J$. Austral. Math. Soc. Ser. A 36 (1984), 279-286.

[15] T. Sherman, 'Representations of Lie algebras by normal operators', Proc. Amer. Math. Soc. 16 (1965), 1125-1129.

[16] I. M. Singer, 'Uniformly continuous representations of Lie groups', Ann. of Math. 56 (1952), 242-247.

[17] J. Tits, 'Algebraic and abstract simple groups', Ann. of Math. 80 (1964), 313-329.

[18] B. L. van der Waerden, 'Stetigkeitssätze für halbeinfache Liesche Gruppen', Math. Z. 36 (1932), 780-786.

\section{Department of Mathematics CP 216}

Université Libre de Bruxelles

Boulevard du Triomphe

B-1050 Brussels

Belgium 